\title{
Dealing with Anxiety: Relationships among Interpersonal Attachment Style, Psychological Wellbeing and Trait Anxiety
}

\author{
Emma E. E. Andrews ${ }^{1} \&$ Richard E. Hicks ${ }^{1}$ \\ ${ }^{1}$ School of Psychology, Bond University, Queensland, Australia \\ Correspondence: Richard E. Hicks, School of Psychology, Bond University, Robina 4229, Queensland, Australia. \\ Tel: 61-7-5595-2580. E-mail: rhicks@bond.edu.au
}

Received: October 16, 2017

Accepted: October 27, 2017

Online Published: November 13, 2017

doi:10.5539/ijps.v9n4p53

URL: http://doi.org/10.5539/ijps.v9n4p53

\begin{abstract}
Anxiety is a major contributor to poor quality mental health for many people in our community, and is a leading cause of presentations at medical and health clinics. Patterns of trait anxiety, or dysfunctional responding, have become ingrained in individuals' approaches to problems they face. Research has shown that psychological wellbeing and interpersonal attachment style are both predictors of trait anxiety. However, the relationships among these variables have not been clarified. The current study sought to determine whether psychological wellbeing mediates the relationship between interpersonal attachment style and trait anxiety, and which of the six psychological wellbeing subscales would contribute most to any mediation effects. A convenience sample of 149 adult participants from South East Queensland, Australia completed a series of online questionnaires including a demographic questionnaire, the Trait Anxiety subscale of the State-Trait Anxiety Inventory (STAI-Form Y2), the Inventory of Parent and Peer Attachment (IPPA), Ryff's Psychological Wellbeing Scale (PWB), and a Social Desirability Scale (SDS-17). Psychological Wellbeing was found to partially mediate the relationship between interpersonal attachment style and trait anxiety. The Positive Relations with Others subscale of the PWB was the only significant sub-scale of the PWB that significantly predicted trait anxiety. Overcoming anxiety appears to be most related in our sample to those who deal better with interpersonal relations. Targeting this aspect in treatment approaches appears most likely to lead to improved outcomes for clients.
\end{abstract}

Keywords: trait anxiety, interpersonal attachment style, psychological wellbeing, parent and peer attachment

\section{Introduction}

High levels of psychological wellbeing enable individuals to enjoy their day-to-day life, form positive perceptions of their social world, and be resilient to life's challenges. But many people at least part of the time run into life issues that are difficult to deal with. Mental illness is one of the primary causes of disability in Australia, affecting individuals' ability to interact with their environment and often leading to significant psychological distress, diminished quality of life, and a reduction in productivity (Bhadra, 2012). One of the main classifications of mental illness in Australia is anxiety. Anxiety-related conditions affect close to three million people, or $11.2 \%$ of the current Australian population (Australian Bureau of Statistics, 2015). It has consistently been reported that one of the strongest predictors for trait anxiety is interpersonal attachment style, and a more recent body of research has investigated the impact of psychological wellbeing on both anxiety and interpersonal attachments. For example, Zhang, Chen, Ran, and Ma (2016) in a study of participants who recorded an avoidant attachment style, found that psychological wellbeing acted as a protective factor for anxiety, depression, and stress. The current research followed this approach and investigated variables that may be able to prevent or reduce the likelihood of individuals developing an anxiety disorder.

Colonnessi and colleagues (2011) recommended that research should investigate how attachment styles affect the development of anxiety conditions, in combination with other factors such as psychological wellbeing. This is so that practitioners, for example, those working in the field of psychopathology, can utilise attachment and wellbeing as variables to inform psychological treatment (Doumen et al., 2012; Schimmenti \& Bifulco, 2013). Utilising attachment-based interventions such as Emotionally Focused Therapy, and Attachment-based Family Therapy (ABFT), in combination with the foundation principles of psychological wellbeing, such as the Broaden-and-Build and Mindfulness frameworks, may have a significant impact on the way in which clinicians 
approach treatment for anxiety disorders (Colonnesi et al., 2011; Jakobsen, Horwood, \& Fergusion, 2011). Investigating the aetiologiy of anxiety conditions may reveal findings that will ultimately enhance and broaden the array of available treatments, and improve quality of life for many individuals (Armbruster, 2008). In the meantime, however, we need to know more about how attachment, wellbeing, and anxiety are related.

The Biopsychosocial model provides a multi-faceted theoretical approach to investigating anxiety that can aid in identifying potential buffers that may assist in prevention and treatment strategies (Schimmenti \& Bifulco, 2013). The model proposes that there are many triggers for anxiety that are categorised into biological, social, and psychological causes that are expressed in cognitive, physical, and psychological symptoms (Jakobse, Horwood, \& Fergusson, 2011). Cognitive symptoms can include decreased concentration, attention deficits, and memory impairment. Physical symptoms involve hyperventilation, trembling, restlessness, and activation of the fight-or-flight mechanism, and psychological symptoms focus on the patterns of cognition related to people's beliefs and self-perception, and how they subsequently perceive their environment (Hassan \& Sahranavar, 2015). In order to conceptualise the development of anxiety disorders from multiple viewpoints, this research utilised the State-Trait Anxiety Inventory (STAI; Spielberger, Gorsuch, Lushene, Vagg, \& Jacobs, 1983) to assess levels of anxiety. The current research utilised trait anxiety (Form Y-2) in relation to interpersonal attachment style and psychological wellbeing. Trait anxiety reflects the way in which an individual responds in anticipation of a threatening situation (Hassan \& Sahranavar, 2015). It is a stable emotional response that does not differ significantly in its presentation in different circumstances. Previous research has shown that individuals who have increased levels of trait anxiety are more likely to be classified as having an anxiety disorder (Breinholst, Esbjørn, \& Reinholdt-Dunne, 2015).

An attachment style developed in infancy is likely to mould all of an individual's interpersonal relationships across their life span (Danquah \& Berry, 2013; Holtfreter, Reisig, \& Turanovic, 2015). Early bonding experiences are a significant predictor of health and wellbeing and when these bonding experiences are less than optimal the relational capacity of a person is impaired, and conditions such as anxiety are seen to develop (Ainsworth, Blehar, Waters, \& Wall, 1978; Stevenson-Hinde, 2007). There are three predominant attachment styles that have emerged in the literature (Ainsworth \& Bell, 1970; Armsden \& Greenberg, 1987): Secure, Insecure-Avoidant, and Insecure-Ambivalent attachment. These attachment styles are based on four anxiety and avoidance dimensions: secure, preoccupied, dismissing, and fearful (Sigelman, Rider, \& De George Walker, 2012). Secure attachment is seen when individuals expect that their attachment figure will be available, helpful, and responsive to their needs; they also achieve a healthy balance of attachment and autonomy, and use their attachment figure as a safe base from which to explore the environment. Insecure-avoidant attachments tend to be more independent of the attachment figure and more self-reliant. They tend to dismiss the importance of relationships and do not seek close proximity to their attachment figure when they are distressed. Insecure-ambivalent attachments often exhibit dependent behaviour, and lack a coherent strategy for meeting attachment needs. Individuals in this category tend to reject their attachment figure when they are distressed, and they worry about abandonment.

Styles of attachment lead to specific internal working models that are mental representations of their social world (Brumariu \& Kerns, 2012). These models present during stressful situations as they lead to different styles of affect regulation in turn implicating psychological development (Schiffrin, 2013). A study by Armbruster (2008) found that infants who had secure attachment styles developed internal working models of relationships in which their needs are met; for infants with insecure attachment styles an internal working model where their needs were barely or never met was developed. Whilst the majority of attachment research focuses on early childhood development, Holtfreter, Reisig, and Turanovic (2015) found that depressive symptoms were lower among older individuals with strong, positive relationships with their spouses and children. This shows how attachment style has a bearing on psychological functioning across the lifespan. One major aspect in the secure-insecure attachment styles is the degree to which the style reflects trust and communication with others or alienation from them. We used the IPPA's measures of trust, communication and alienation to calculate an interpersonal attachment style score that reflected secure attachment at one end and insecure attachment at the other (see Method).

Psychological wellbeing is conceptualised as a measure of individuals' evaluations of their own experiences, which is comprised according to Ryff (1995) of three main elements: life satisfaction, positive affect, and negative affect. Psychological interventions that are based upon the principles of psychological wellbeing, such as aiming to cultivate positive emotions, feelings, behaviours, and cognitions, have been found to significantly improve overall wellbeing and reduce the presentation of mental health conditions (Sin \& Lyubomirsky, 2009). The recent shift in focus from the traditional disease model prevalent in psychology, to a holistic and positive 
approach to mental health has meant an increased interest in empirical research that investigates psychological wellbeing. The success of positive psychology in psychotherapy, such as using the Broaden-and-Build model, is evidenced through patients reporting higher levels of psychological wellbeing after participation in these programs. Evaluating the extent to which psychological wellbeing can buffer the effects of a negative insecure attachment style could lead to advances in the treatment of aspects of anxiety disorders (by emphasising skills development in relevant psychological wellbeing attributes).

Individuals with high levels of psychological wellbeing perceive their social world in a more receptive and attentive way than do others (Weinsten, Brown, \& Ryan, 2009). Having a broadened state of awareness facilitates positive interpretations of stressful life events, which can lead to substantially reduced distress (Garland, Gaylord, \& Fredrickson, 2011). The PWB was selected for the current study as it assesses overall wellbeing but also assesses six subscales: autonomy, positive relations with others, personal growth, environmental mastery, purpose in life and self-acceptance.

Much research has investigated the predisposing factors for internalising problems such as anxiety. In a cohort of undergraduate Japanese students it was seen that the dimensions of psychological wellbeing acted as protective factors against generalised anxiety disorder (Takebayashi, Tanaka, Sugiura, \& Sugiura, 2017). Jakobsen, Horwood, and Fergusson (2011) also found that interpersonal relationships and attachment styles significantly impacted the development of anxiety disorders in early adulthood.

Armsden and Greenberg (1987) had identified similar findings and discussed how secure attachment styles were able to provide a protective function against the later development of physical and mental illness. Armbruster (2008) also identified that a secure attachment style is significantly negatively correlated with all types of anxiety. Similarly, Lecompte, Mos, Cyr, and Pascuzzo (2014) found that insecure attachment is a risk factor for the development of internalising disorders such as anxiety. In another study Raja, McGee, and Stanton (1992) found that in a sample of $935 \mathrm{New}$ Zealand adolescents, the mean anxiety scores were similar in individuals showing secure or insecure styles but drew an overall conclusion that secure and stable attachment styles act as a buffer against adolescent anxiety.

Interpersonal attachment styles are also important predictors of psychological wellbeing (Cropanzo \& Dasborough, 2015). Internal working models that are developed with different styles of attachment help explain emotional distress experienced when attachment relationships are disrupted (Didge, Daly, Huyton, \& Sanders, 2012). Davis, Morris, and Drake (2016) identified that wellbeing assessed by measures of depression, anxiety, and stress were strongly associated with an individual's attachment style, and that attachment anxiety showed a predictive capacity for wellbeing. LaGuardia, Ryan, Crouchman, and Deci (2001) found evidence in a sample of 136 university students that individuals feel more capable of satisfying their basic psychological needs when they feel secure within their attachments. Raja, McGee, and Stanton (1992) found a significant relationship between psychological wellbeing and attachment styles, such that those who were securely attached reported significantly fewer negative emotions and higher self-regard in comparison to those who were insecurely attached. Therefore, examining relationships among all three (attachment style, anxiety and psychological wellbeing) could yield information useful in practical interventions to reduce anxiety.

Proponents of positive psychology argue in favour of emphasising wellbeing interventions in the treatment of anxiety disorders (De Beurs, Beekman, Van Balkom, Deeg, Van Dyck, \& Van Tillburg, 1999). Typically a significant negative correlation between anxiety and psychological wellbeing is found in research. Piet, Würtzen, and Zacharie (2012) used a sample of oncology patients to assess the efficacy of wellbeing programs (i.e., Mindfulness Based Therapy, MBT) in reducing the symptoms of anxiety. This psychosocial approach to treating internalising disorders aimed to foster positive emotions and focus on psychological wellbeing rather than psychological dysfunction. Results found that these therapeutic approaches were effective in reducing symptoms of anxiety, as they targeted reduction of rumination and emotional avoidance, which are considered to be maintenance factors across all types of anxiety disorders. A meta-analysis conducted by Chiesa and Seretti (2009) found that focusing on an individual's psychological wellbeing helped to increase compliance with treatment, and lead to improvement in reducing trait anxiety. In a similar analysis, Seligman, Ernst, Gillham, Reivich, and Linkins (2009) observed that clinicians who focused on wellbeing helped prevent anxiety symptoms progressing into a diagnosis of an anxiety disorder.

\subsection{The Present Study}

Based on previous empirical research the three overall variables (attachment style; psychological wellbeing; anxiety) tend to be significantly correlated; however, the literature focuses on the variables separately rather than how all three interact. We investigated all three together hypothesising (1) that psychological wellbeing (as 
measured by the PWB: Ryff, 1995) would mediate the relationship between interpersonal attachment style (as measured by the IPPA: Armsden \& Greenberg, 1987) and trait anxiety (as measured by the STAI: Spielberger et al., 1983).

The subscales of psychological wellbeing were also investigated to see whether there were specific traits that may contribute to the mediation more than others. We explored these relationships in a contemporary Australian sample, hypothesising; (2) that each of the six subscales of the PWB: Environmental Mastery, Personal Growth, Autonomy, Positive Relations with Others, Self-Acceptance, and Purpose in Life, would add significant unique variance in the interpersonal attachment style/anxiety relationship.

\section{Method}

\subsection{Participants}

A convenience sample of 204 participants was recruited to participate in the research. Fifty-five of these participants were removed from analysis due to missing data, and the final analysis included 149 participants. Within the final sample 37 participants were male (24.8\%), and $112(75.2 \%)$ were female. Participant ages ranged from 17 to 64 years, with a mean age of 25.62 years $(S D=10.75)$. Data assessing attachment style was split into two categories (Secure; Insecure). Insecure-Avoidant and Insecure-Ambivalent styles were combined to yield near equal cell sizes. The Secure Attachment group contained 74 participants-17 male (23\%) and 57 female $(77 \%)$ with age range 17 to 64 with a mean age of 26.81 years $(S D=12.08)$. The Insecure Attachment group contained 75 participants - 20 male (26.7\%) and 55 female (73.3\%) with age range 18 to 58 with a mean age of 24.44 years $(S D=9.18)$. The Secure and Insecure groups were thus seen to be similar on the age and gender distributions.

\subsection{Materials}

The series of measures described below were administered online to all participants.

\subsubsection{Demographics Scale}

Participants answered questions about their age, gender, highest level of education, and employment status.

\subsubsection{The Inventory for Parent and Peer Attachment (IPPA: Armsden \& Greenberg, 1987)}

The IPPA was developed in relation to the three main attachment styles detailed by Ainsworth (1979): the version we used in our study contained 75 items related to attachment to Mother, Father, and Peers; these items assessed three interpersonal attachment factors: Trust, Communication, and Alienation (Gullone \& Robinson, 2005). Items are presented on a five-point Likert-type scale with response categories ranging from (1) "Not true" to (5) "Always true". Participants were asked to rate a statement based on its relevance to them; an example of a mother-child item is, "My mother respects my feelings", an example of a father-child item is, "My father accepts me as I am", and an example of a peer related item is, "My friends care about how I am feeling". Good internal consistency for this measure has been reported with coefficients for Cronbach's alpha reaching 0.91 for all subscales (Gorrese \& Ruggieri, 2012). The current sample yielded similar high level Cronbach alpha coefficients.

To calculate a total attachment score, the three factors (Trust, Communication, and Alienation) were added together to create a total score combining each scale (Mother, Father, and Peer). The Alienation factor was reverse-scored as well as five-items from the Trust, and Communication factor questions. A total attachment score was then created by combining the Mother, Father, and Peer attachment scores. The IPPA has been consistently reported as a useful tool for the assessment of attachment and psychological security (Gorrese \& Ruggierei, 2012). Adequate convergent validity has also been established (e.g., Pace, San Martini, \& Zavattini, 2011).

\subsubsection{The State-Trait Anxiety Inventory (STAI: Spielberger, Gorsuch, Lushene, Vagg, \& Jacobs, 1983)}

For the purpose of this research, only the Trait Anxiety (Form Y2) subscale was used to assess participants' level of anxiety. This subscale contains 20 -items that are presented on a four-point Likert type scale with response categories ranging from (1) "Almost Never" to (4) "Almost Always". Multiple items are reverse scored and scores can range from 20 to 80, with greater scores indicating increased anxiety. The T-anxiety Inventory (SAI Form Y-2) is designed to assess how a participant feels generally (Hassan \& Sahranavar, 2015). An example of an item from this form is, "I worry too much over something that really doesn't matter". Internal consistency for this form is considered good-to-excellent with coefficients for Cronbach's Alpha reported between 0.86 and 0.95 (Hill, Musso, Jones, Pella, \& Gouvier, 2012). The alpha level as determined in the current analysis was similar (.92). 


\subsubsection{The Ryff Psychological Wellbeing Scale (PWB: Ryff, 1995)}

The PWB is a theoretically grounded self-report scale designed to measure six dimensions of psychological wellbeing: Autonomy, Environmental Mastery, Personal Growth, Positive Relations with Others, Purpose in Life, and Self-Acceptance (Ryff \& Keyes, 1995). The scale used in this study is the PWB medium form which contains 54-items that are presented on a six-point Likert-type scale from (1) "Strongly Disagree" to (6) "Strongly Agree". An example of an item is, "I enjoy making plans for the future and working to make them a reality". Higher scores on each scale indicate greater psychological wellbeing. Each subscale contains nine items with a mix of positive and negatively worded statements. For the purpose of the first hypothesis, a composite total score adding all items was used to evaluate a participants' psychological wellbeing. For the second hypothesis the six subscales of the PWB were analysed separately. Internal consistency was excellent yielding a Cronbach alpha's coefficient of .92 for the full scale. The internal consistency for the six subscales presented with adequate psychometric properties with Cronbach's alpha ranging from 0.70 to 0.84 .

\subsubsection{The Social Desirability Scale-17 (SDS-17: Stober, 1999)}

The SDS-17 is a revised scale that assesses whether a participant appears to be answering questions to make a favourable impression. The SDS-17 is a shorter version of the 33-item SDS developed by Marlowe and Crowne (1960). The version used in the current research contained 16-items, as research had recommended deletion of one item (Lambert, Arbuckle, \& Holden, 2016). The scale is formatted dichotomously, and participants are asked to respond to a statement as true or false. An example of a socially desirable true item is, "I never hesitate to help someone in case of an emergency". Higher scores represent participants who select responses that tend to present them in a favourable light. The internal consistency value for this measure, as determined in the current analysis, was sound with Cronbach's alpha being 0.69.

\subsection{Procedure}

Once ethical approval was received from the University Human Research Ethics Committee, potential convenience sample respondents were approached to complete the series of measures relating to anxiety, attachment, and wellbeing, using the online platform Psych Data. An explanatory statement was displayed on the first page that detailed the issues of confidentiality, anonymity, risk, and an overview of the research project. Once acceptance of these conditions had occurred the participants then completed the questionnaires in the order: demographics, STAI, IPPA, PWB, and SDS-17. Several other measures were also included in the online questionnaire set; however, they were not used in this reported research. Invitations were distributed through online networks, where a hyperlink to the questionnaires was attached. Participants were recruited through the university student research system, social media, email, and other online avenues. Participants were asked to share the invitation to participate with their contacts to expand the participant pool through the process of virtual snowballing (following Balter \& Brunet, 2012). As an incentive, after completion of the questionnaires participants were offered one credit point if they came from a University Undergraduate Psychology participant pool. Participation in the research was voluntary and participants were informed they could cease participation at any point without penalty.

\section{Results}

Analyses were conducted using SPSS Statistics 22. As indicated above, due to systematic missing data, 55 cases were removed leaving the final sample as $N=149$. This sample size was deemed adequate, as tested against Tabachnik and Fidell's (2001) model for power.

\subsection{Preliminary Analyses}

Table 1 presents the means, standard deviations, and inter-correlations, for scores on Trait Anxiety, Psychological Wellbeing and Interpersonal Attachment Style.

Table 1. Summary of pearson product moment correlations, means, and standard deviations ( $\mathrm{N}=149)$

\begin{tabular}{cccccc}
\hline Variable & 1 & 2 & 3 & $M$ & $S D$ \\
\hline 1. TA & - & & & 42.03 & 11.29 \\
2. PWB & $-.44^{* *}$ & - & 227.33 & 31.66 \\
3. IAS & $-.28^{* *}$ & $.24^{* *}$ & - & 254.11 & 34.72 \\
\hline
\end{tabular}

Note. $M=$ Mean; $S D=$ Standard Deviation; $T A=$ Trait Anxiety; $P W B=$ Psychological Wellbeing; $I A S=$ Interpersonal Attachment Style. ${ }^{*} p<.05, * * p<.01, * * * p<.001$. 


\subsection{Mediation Analyses: Basic Model}

Baron and Kenny's (1986) first step in mediation requires that the predictor variable be correlated significantly with the outcome variable. Interpersonal attachment style was significantly (negatively) correlated with trait anxiety. Cohen's (1984) conventions indicate that the relationship had a small effect size, quantifying the strength of the relationship. Baron and Kenny's (1986) second step requires that the predictor variable is correlated with the mediator variable. Interpersonal attachment style was significantly (positively) correlated with psychological wellbeing. With regards to Cohen's (1984) conventions, this relationship also had a small effect size. The third step requires that the mediator variable affect the outcome variable in a significant way. Psychological wellbeing was significantly (negatively) correlated with trait anxiety. This relationship also yielded a small-to-medium effect size (Cohen, 1984).

\subsection{Mediated Multiple Regression Analysis}

To examine whether psychological wellbeing mediated the relationship between interpersonal attachment style and trait anxiety, a simple regression, a hierarchical multiple regression, and a Sobel test were conducted. The first analysis showed path $a$ of the indirect effect to be significant-the analysis revealed interpersonal attachment style accounted for a statistically significant amount of variance in psychological wellbeing, $R^{2}=.04$, Adjusted $R^{2}=.03, F(1,146)=5.37, p=.022$. The coefficient for interpersonal attachment style to psychological wellbeing (path $a$ ) was significant $(\beta=-.19, p=.022$ ).

To examine the relationship between the mediator (each subscale in turn of the PWB) and the outcome variable (TA: path $b, c$, and direct effect), a hierarchical multiple regression analysis was performed. In this analysis, Age, Gender, Education, and scores on the Social Desirability Scale were entered at Step 1 to control for potential covariance. The predictor variable (interpersonal attachment style) was then entered at Step 2, and the mediator variable (psychological wellbeing) was entered at Step 3. This tested path $b$ and path $c^{\prime}$ in the mediation model in addition to path $c$, which showed the total effect of the unmediated model (Baron \& Kenny, 1986). Table 2 displays the unstandardized regression coefficients, and associated $95 \%$ confidence intervals, in addition to the standardised regression coefficients and $\mathrm{R}_{\text {change. }}^{2}$

Table 2. Hierarchical multiple regression analysis predicting trait anxiety from interpersonal attachment style and psychological wellbeing $(\mathrm{N}=149)$

\begin{tabular}{|c|c|c|c|c|c|}
\hline Variables & $\Delta \mathrm{R}^{2}$ & $\beta$ & B & SE B & $95 \%$ for $\mathrm{CI}$ \\
\hline Step 1 & $.08^{*}$ & & & & \\
\hline Constant & & & 33.90 & 5.57 & {$[22.88,44.94]$} \\
\hline Age & & -.15 & -0.14 & 0.09 & {$[-0.33,0.04]$} \\
\hline Gender & & $.22 *$ & 5.80 & 2.30 & {$[1.24,10.36]$} \\
\hline Education & & .02 & 0.20 & 1.05 & {$[-1.88,2.29]$} \\
\hline SDS-17 & & .04 & 0.13 & 0.33 & {$[-0.52,0.77]$} \\
\hline Step 2 & $.06^{* *}$ & & & & \\
\hline Constant & & & 39.38 & 1.28 & {$[36.81,41.87]$} \\
\hline IAS & & $.25 * *$ & 5.70 & 1.81 & {$[2.13,9.28]$} \\
\hline Step 3 & $.15 * * *$ & & & & \\
\hline Constant & & & 72.67 & 6.45 & {$[59.92,85.41]$} \\
\hline IAS & & $.18^{*}$ & 4.03 & 1.70 & {$[0.67,7.38]$} \\
\hline PWB & & $-.39 * * *$ & -0.14 & 0.03 & {$[-0.20,-0.09]$} \\
\hline Total $\mathrm{R}^{2}=.29$ & & & & & \\
\hline
\end{tabular}

Note. SE B=Standard Error of Unstandardised Coefficient; $C I=$ Confidence Interval.

${ }^{*} p<.05, * * p<.01, * * * p<.001$.

As can be seen in Table 2, when potential covariates (Age, Gender, Education, and SDS-17 Scores) were entered at Step 1 , they accounted for a statistically significant $8 \%$ of the variance in trait anxiety, $\mathrm{R}_{\text {change }}^{2}=.08, F_{\text {change }}(4$, 
146) $=2.59, p=0.40$. When interpersonal attachment style was entered at Step 2, it accounted for a statistically significant additional $6 \%$ of the variance in trait anxiety, $\mathrm{R}_{\text {change }}=.06, F_{\text {change }}(1,145)=9.92, p=0.002$. At this step, trait anxiety was significantly positively predicted by attachment style $(\beta=.25, p=.002)$, such that a $1 S D$ increase in attachment style was related to a $.25 S D$ increase in anxiety. This assessed the direct effect (path $c$ ), indicating that there was an effect to be mediated.

After controlling for the effects of the covariates and attachment style, psychological wellbeing was entered at Step 3 and accounted for $15 \%$ of the variance in trait anxiety over and above interpersonal attachment style $\mathrm{R}_{\text {change }}^{2}=.15, F_{\text {change }}(1,143)=27.64, p<0.001$. At this step (path $b$ ), the coefficient for the mediator variable (psychological wellbeing) was significant $(\beta=-.39, p<.001)$, such that a $1 S D$ increase in psychological wellbeing was related to a $39 S D$ decrease in trait anxiety. At this step again (path $c^{\prime}$ ), the direct path in the mediated model was still significant.

Whilst the regression coefficient for interpersonal attachment style decreased marginally it was still significantly different from zero $(\beta=.18, p=.019)$. This is partial mediation, as although the regression coefficient has decreased, it is still significant at Step 3. The results of a Sobel test (Sobel, 1989) also highlighted that the decrease in the regression coefficient was significant $(z=2.13, p=0.03)$, indicating that a significant indirect effect is evident. The unmediated and mediated models are presented in Figure 1.

a) Unmediated model

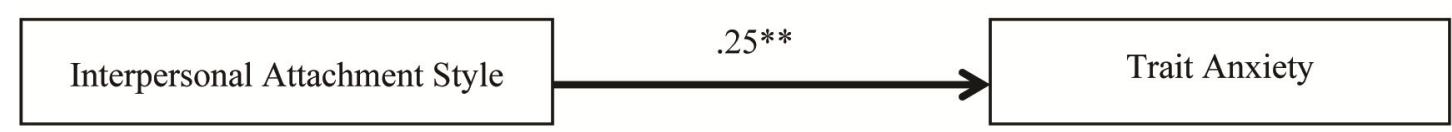

b) Mediated Model

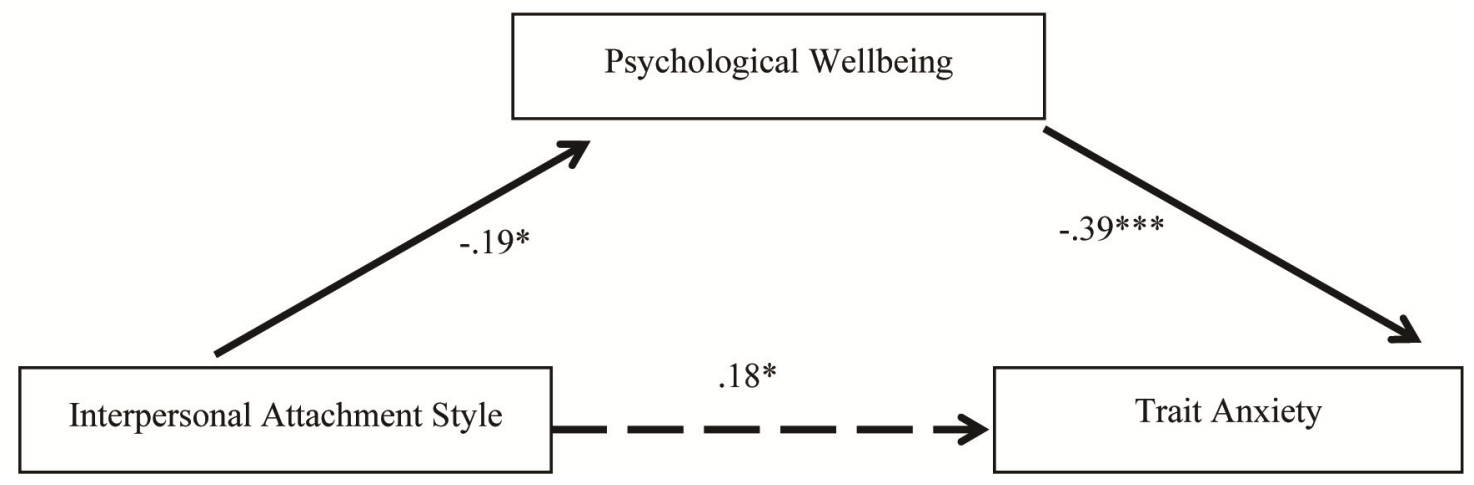

Figure 1. The relationship between interpersonal attachment style and trait anxiety, with confirmed partial mediation by psychological wellbeing

Note. ${ }^{*} p<.05, * * p<.01, * * * p<.001$.

\subsection{Follow-Up Analyses (Hypothesis 2 on the Psychological Wellbeing Subscales)}

Following a significant finding in the mediation between Interpersonal Attachment Style, Psychological Wellbeing, and Trait Anxiety, a series of follow-up analyses were run to examine whether all or certain subscales of the PWB affected Trait Anxiety. In accordance with Cohen's conventions (1994), all six subscales had small-to-medium effect sizes and were significantly negatively related to trait anxiety. Autonomy correlated significantly with Trait Anxiety ( $\mathrm{r}=-0.46$ ), Environmental Mastery correlated -0.45 ; Personal Growth -0.30 ; Positive Relations with Others -0.52; Purpose in Life -0.45; and Self-Acceptance -0.33.

Hierarchical Multiple Regression Analysis. A six-step hierarchical multiple regression analysis was conducted with Trait Anxiety as the outcome variable. The six PWB variables were entered into the regression equation based on a descending order of effect sizes. The unstandardized regression coefficients and associated $95 \%$ confidence intervals in addition to the standardised regression coefficients and $\mathrm{R}^{2}$ for the model, as well as $\mathrm{R}_{\text {change }}^{2}$ can be seen in Table 3 .

At Step 1 of the Hierarchical Multiple Regression Analysis, Positive Relations with Others accounted for a significant $27 \%$ of the variance in Trait Anxiety, $\mathrm{R}_{\text {change }}^{2}=.27, F_{\text {change }}(1,146)=53.74, p<.001$. This meant that 
higher scores on this subscale were related to lower scores on Trait Anxiety $(\beta=-.52, p<.001)$, and where there was a $1 S D$ increase in Positive Relations with Others; there was a.52SD decrease in Trait Anxiety.

Table 3. Hierarchical multiple regression analysis predicting trait anxiety from subscales of psychological wellbeing $(\mathrm{N}=151)$

\begin{tabular}{cccccc}
\hline Variables & $\Delta \mathrm{R}^{2}$ & $\beta$ & $\mathrm{B}$ & $\mathrm{SE}$ B & $95 \%$ for CI \\
\hline Step 1 & $.27^{* * *}$ & -.52 & 78.66 & 5.03 & {$[68.72,88.60]$} \\
Constant PRO & & & -0.99 & 0.14 & {$[-1.26,-0.73]$} \\
Step 2 & .01 & -.15 & 80.72 & 5.23 & {$[70.39,91.06]$} \\
Constant A & & -0.27 & 0.19 & {$[-0.65,0.11]$} \\
Step 3 & .002 & -.08 & 81.20 & 5.30 & {$[70.72,91.67]$} \\
Constant PL & & -0.12 & 0.21 & {$[-0.54,0.29]$} \\
Step 4 & .02 & -.18 & 84.31 & 5.54 & {$[73.36,95.24]$} \\
Constant EM & & -0.12 & 0.21 & {$[-0.80,0.04]$} \\
Step 5 & .02 & .24 & 82.97 & 5.53 & {$[72.04,93.90]$} \\
Constant SA & & 0.38 & 0.20 & {$[-0.01,0.76]$} \\
Step 6 & .004 & .10 & 80.09 & 6.31 & {$[67.62,92.56]$} \\
Constant PG & & 0.21 & 0.22 & {$[-0.22,0.64]$} \\
\hline
\end{tabular}

Note. $S E B=$ Standard Error of Unstandardised Coefficient; $C I=$ Confidence Interval; A=Autonomy; EM=Environmental Mastery; $\mathrm{PG}=$ Personal Growth; $\mathrm{PRO}=$ Positive Relations with Others, $\mathrm{PL}=$ Purpose in Life; SA=Self Acceptance.

${ }^{*} p<.05,{ }^{* *} p<.01, * * * p<.001$.

No other subscales accounted for any additional significant variance in Trait Anxiety. As Table 3 shows, with consideration to unique variance, Positive Relations with Others contributed $27 \%$ of the variance in Trait Anxiety, which was significant. Autonomy, Purpose in Life, Environmental Mastery, Self-Acceptance, and Personal Growth did not contribute significant amounts of unique variance beyond that already accounted for by Positive relations with others, with each contributing respectively just $1 \%, .2 \%, 1.6 \%, 1.8 \%$ and $.4 \%$ in predicting trait anxiety. There was no variance that was shared by all six variables. In summary, the analysis revealed that the Positive Relations with Others subscale of the Psychological Wellbeing Scale contributed most to the mediation between Interpersonal Attachment Style and Trait Anxiety and was the main aspect of importance in the relationships among interpersonal attachment style, psychological wellbeing and trait anxiety expression.

\section{Discussion}

The aim of the current research was to investigate the association between interpersonal attachment styles and the development of anxiety disorders, and whether this relationship could be mediated by psychological wellbeing. Previous research had found tangible links between all three variables (e.g., Cropanzo \& Dasborough, 2015); however, there was insufficient evidence linking these variables in a mediation model. In the process of examining the mediation role of psychological wellbeing we noted similarities in our research findings to those of previous studies that had examined separately the relationships among attachment styles, anxiety and wellbeing. For example, our study found that interpersonal attachment style (secure attachment style as measured by the combination of scores on Trust, Communication and Alienation) was significantly associated with trait anxiety - a necessary step in carrying out the mediation analyses. This finding was consistent with and confirms previous research studies and reports such as those of Armbruster (2008), Holtfreter, Reisig, and Turanovic (2015), Lecompte, Mos, Cyr, and Pascuzzo (2014), and Raja, McGee, and Stanton (1992).

Our study also examined relationships between psychological wellbeing and attachment, and psychological wellbeing and trait anxiety. For our study to proceed it was necessary that these relationships were significant (correlated significantly). This was the case and again was consistent with the previous studies that had examined these relationships separately. For example, previous studies that our results confirmed in regard to the attachment style and wellbeing relationship included those of Crpanzo and Dasborough (2015), Davis, Morris, 
and Drake (2016), Raja, McGee, and Stanton (1992), and Zhang, Chen, Ran, and Ra (2016). Further we confirmed previously identified significant negative relationships between wellbeing and anxiety, as in studies by researchers such as Piet, Wurtzen, and Zacharie (2012), Sin and Lyubomirsky (2009), and Takebayashi, Tanaka, Sugiura, and Suguira (2017).

The current study however, examined two hypotheses incorporating the three elements in the one study (or series). Firstly, that psychological wellbeing would mediate the relationship between interpersonal attachment style and trait anxiety; and secondly, that each subscale of the PWB would add unique variance at its own point of entry into the regression equation.

The first hypothesis was supported; psychological wellbeing mediated the relationship between interpersonal attachment style and trait anxiety but did so partially (that is both interpersonal attachment style and psychological wellbeing-negatively-together contributed to trait anxiety). Psychological wellbeing is an important mediator in the relationship between attachment style and anxiety but after allowing for the contribution from psychological wellbeing the effects of attachment style (trust, communication and alienation) still apply. Few studies appear to have previously examined mediators among these three attributes and the results indicate the importance of giving attention to improving both attachment style and wellbeing skills and attributes when aiming to treat trait anxiety.

The second hypothesis examined each of the six subscales within psychological wellbeing and in combination (regression) to identify the contributions made by the scales. As expected there were significant negative correlations from each of the subscales in relation to trait anxiety, mirroring the total psychological capital core in relation to trait anxiety. So there was partial support for the hypothesis. However, when all six scales were included in the regression equations associated with mediation only one of the six scales of the PWB added significant unique variance into the regression equation. Consistent with the literature, the Positive Relations with Others subscale significantly predicted trait anxiety. The remaining five facets contributed additional variance but this was not significant. The implication of this finding is that it further confirms the relationship between attachment style and psychological wellbeing (especially the value of positive relationships with others); reinforcing the importance of utilising both aspects of attachment style and relationship building when treating an anxiety disorder.

\section{Limitations}

Although both hypotheses were supported, there are some limitations that may have inhibited more significant results. The exclusive use of self-report inventories may have lead to biased responding. Even though a validity check in the form of the Social Desirability Scale (SDS-17) was employed, a small percentage did not complete this measure. This was due to this measure being last in the questionnaire order and in future it would be better to counterbalance the forms. As with most empirical investigations, the issue of generalizability was another limitation. The majority of respondents were drawn from a population of students who received class credit for their participation. It is therefore reasonable to assume that this was a fairly high-functioning group of individuals, and the potential ranges apparent within the trait anxiety category could be reduced.

\section{Conclusion}

Despite these limitations, this research has produced empirical evidence that both supports and extends upon previous literature, as well as identifying significant relationships between the variables. As a preliminary examination into the relationships among attachment style, trait anxiety, and psychological wellbeing, this research may act as a catalyst for further empirical investigation. By demonstrating the mediational model between attachment style, psychological wellbeing and trait anxiety the current research project supports the need to include strategies that improve psychological wellbeing in psychotherapy along with using a client's attachment style as a way to inform the focus of treatment.

A significant contribution from the current study is the affirmation of the importance of building improved relationships with others. While it had been identified in previous research that interpersonal attachment style was related (negatively) to trait anxiety expression, and that psychological wellbeing had also been seen to be significantly (negatively) related to trait anxiety expression, the pre-eminent contribution of made by positive relationships with others had not been highlighted. This finding confirms the likely value of emphasising skills improvement in personal relationships for those aiming to deal with their trait anxiety, and of course confirms practitioner efforts to help their clients improve their skills in this area. 


\section{References}

Ainsworth, M., \& Bell, S. (1970). Attachment, Exploration, and Separation: Illustrated by the Behavior of One-Year-Olds in a Strange Situation. Child Development, 41(1), 49. https://doi.org/10.2307/1127388

Ainsworth, M., Blehar, M., Waters, E., \& Wall, S. (1978). Patterns of attachment (pp. 160-186). Hillsdale, N.J.: Lawrence Erlbaum Associates.

Armbruster, E. (2008). Attachment and bonding: Correlations between relation and anxiety among adult college students. University of New Mexico.

Armsden, G., \& Greenberg, M. (1987). The inventory of parent and peer attachment: Individual differences and their relationship to psychological well-being in adolescence. $J$ Youth Adolescence, 16(5), 427-454. https://doi.org/10.1007/BF02202939

Australian Bureau of Statistics. (2015, July). 4364.0.55.001-National Health Survey: First Results, 2014-15. Retrieved May 6, 2016, from http://www.abs.gov.au/ausstats/abs@.nsf/Lookup/by\%20Subject/4364.0.55.001 201415 Main\%20Feature s $\sim$ Mental\%20and\%20behavioural\%20conditions $\sim 32$

Balter, F., \& Brunet, I. (2012). Social research 2.0: Virtual snowballing sampling method using Facebook. Internet Research, 22(1), 57-74. https://doi.org/10.1108/10662241211199960

Baron, R., \& Kenny, D. (1986). The moderator-mediator variable distinction in social psychological research: Conceptual, strategic, and statistical considerations. Journal of Personality and Social Psychology, 51(6), 1173-1182. https://doi.org/10.1037/0022-3514.51.6.1173

Bhadra, M. (2012). Mental Health \& Mental Illness: Our Responsibility. Health Renaissance, 10(1). https://doi.org/10.3126/hren.v10i1.6014

Breinholst, S., Esbjørn, B., \& Reinholdt-Dunne, M. (2015). Effects of attachment and rearing behavior on anxiety in normal developing youth: A mediational study. Personality and Individual Differences, 81, 155-161. https://doi.org/10.1016/j.paid.2014.08.022

Brumariu, L., \& Kerns, K. (2012). Pathways to Anxiety: Contributions of Attachment History, Temperament, Peer Competence, and Ability to Manage Intense Emotions. Child Psychiatry Hum Dev, 44(4), 504-515. https://doi.org/10.1007/s10578-012-0345-7

Chiesa, A., \& Seretti, A. (2009). Mindfulness-Based Stress Reduction for Stress Management in Healthy People: A Review and Meta-Analysis. The Journal of Alternative And Complementary Medicine, 15(5), 593-600. https://doi.org/10.1089/acm.2008.0495

Cohen, J. (1994). The earth is round $(\mathrm{p}<.05) . \quad$ American Psychologist, 49(12), 997. https://doi.org/10.1037/0003-066X.49.12.997

Colonnesi, C., Draijer, E., Jan J. M., Stams, G., Van der Bruggen, C., Bögels, S., \& Noom, M. (2011). The Relation between Insecure Attachment and Child Anxiety: A Meta-Analytic Review. Journal of Clinical Child \& Adolescent Psychology, 40(4), 630-645. https://doi.org/10.1080/15374416.2011.581623

Cropanzano, R., \& Dasborough, M. (2015). Dynamic models of well-being: Implications of affective events theory for expanding current views on personality and climate. European Journal of Work and Organizational Psychology, 24(6), 844-847. https://doi.org/10.1080/1359432X.2015.1072245

Danquah, A., \& Berry, K. (2013). Attachment theory in adult mental health. London: Routledge.

Davis, T., Morris, M., \& Drake, M. (2016). The moderation effect of mindfulness on the relationship between adult attachment and wellbeing. Personality and Individual Differences, 96, 115-121. https://doi.org/10.1016/j.paid.2016.02.080

De Beurs, E., Beekman, A., Van Balkom, A., Deeg, D., Van Dyck, R., \& Van Tilburg, W. (1999). Consequences of anxiety in older persons: Its effect on disability, well-being and use of health services. Psychological Medicine, 29(3), 583-593. https://doi.org/10.1017/S0033291799008351

Didge, R., Daly, A., Huyton, J., \& Sanders, L. (2012). The challenge of defining wellbeing. International Journal of Wellbeing, 2(3), 222-235. https://doi.org/10.5502/ijw.v2i3.4

Doumen, S., Smits, I., Luyckx, K., Duriez, B., Vanhalst, J., Verschueren, K., \& Goossens, L. (2012). Identity and perceived peer relationship quality in emerging adulthood: The mediating role of attachment-related emotions. Journal of Adolescence, 35(6), 1417-1425. https://doi.org/10.1016/j.adolescence.2012.01.003 
Gorrese, A., \& Ruggieri, R. (2012). Peer Attachment: A Meta-analytic Review of Gender and Age Differences and Associations with Parent Attachment. $J$ YouthAdolescence, 41(5), 650-672. https://doi.org/10.1007/s10964-012-9759-6

Gullone, E., \& Robinson, K. (2005). The Inventory of Parent and Peer Attachment? Revised (IPPA-R) for children: A psychometric investigation. Clinical Psychology \& Psychotherapy, 12(1), 67-79. https://doi.org/10.1002/cpp.433

Hassan, S., \& Sahranavar, M. (2015). Investigation of Construct Validity of State Anxiety Inventory among Iranian 8th Grade Students. Asian Journal of Applied Sciences, 8(1), 63-70. https://doi.org/10.3923/ajaps.2015.63.70

Hill, B., Musso, M., Jones, G., Pella, R., \& Gouvier, W. (2012). A Psychometric Evaluation of the STAI-Y, BDI-II, and PAI Using Single and Multifactorial Models in Young Adults Seeking Psychoeducational $\begin{array}{llll}\text { Evaluation. Journal of Psychoeducational Assessment, 31(3), 300-312. } & \text {. }\end{array}$ https://doi.org/10.1177/0734282912462670

Holtfreter, K., Reisig, M., \& Turanovic, J. (2015). Depression and infrequent participation in social activities among older adults: The moderating role of high-quality familial ties. Aging \& Mental Health, 21(4), 379-388. https://doi.org/10.1080/13607863.2015.1099036

Jakobsen, I., Horwood, L., \& Fergusson, D. (2011). Childhood Anxiety/Withdrawal, Adolescent Parent-Child Attachment and Later Risk of Depression and Anxiety Disorder. Journal Of Child And Family Studies, 21(2), 303-310. https://doi.org/10.1007/s10826-011-9476-x

La Guardia, J., Ryan, R., Couchman, C., \& Deci, E. (2000). Within-person variation in security of attachment: A self-determination theory perspective on attachment, need fulfillment, and well-being. Journal of Personality and Social Psychology, 79(3), 367-384. https://doi.org/10.1037/0022-3514.79.3.367

Laible, D., Carlo, G., \& Rafaelli, M. (1999). The Differential Relations of Parent and Peer Attachment to Adolescent Adjustment. Journal of Youth and Adolescence, 29(1), 45-59. https://doi.org/10.1023/A:1005169004882

Lambert, C., Arbuckle, S., \& Holden, R. (2016). The Marlowe-Crowne Social Desirability Scale outperforms the BIDR Impression Management Scale for identifying fakers. Journal of Research in Personality, 61, 80-86. https://doi.org/10.1016/j.jrp.2016.02.004

Lecompte, V., Moss, E., Cyr, C., \& Pascuzzo, K. (2014). Preschool attachment, self-esteem and the development of preadolescent anxiety and depressive symptoms. Attachment \& Human Development, 16(3), 242-260. https://doi.org/10.1080/14616734.2013.873816

Naragon-Gainey, K., Watson, D., \& Markon, K. (2009). Differential relations of depression and social anxiety symptoms to the facets of extraversion/positive emotionality. Journal of Abnormal Psychology, 118(2), 299-310. https://doi.org/10.1037/a0015637

Raja, S., McGee, R., \& Stanton, W. (1992). Perceived attachments to parents and peers and psychological well-being in adolescence. $J$ Youth Adolescence, 21(4), 471-485. https://doi.org/10.1007/BF01537898

Ryff, C. (1995). Psychological Well-Being in Adult Life. Current Directions in Psychological Science, 4(4), 99-104. https://doi.org/10.1111/1467-8721.ep10772395

Schiffrin, H. (2013). Positive Psychology and Attachment: Positive Affect as a Mediator of Developmental Outcomes. Journal of Child And Family Studies, 23(6), 1062-1072. https://doi.org/10.1007/s10826-013-9763-9

Schimmenti, A., \& Bifulco, A. (2013). Linking lack of care in childhood to anxiety disorders in emerging adulthood: The role of attachment styles. Child And Adolescent Mental Health, 20(1), 41-48. https://doi.org/10.1111/camh.12051

Sigelman, C., Rider, E., \& De George-Walker, L. (2012). Life span human development. South Melbourne, Vic.: Cengage Learning.

Sin, N., \& Lyubomirsky, S. (2009). Enhancing well-being and alleviating depressive symptoms woth positive psychology interventions: A practice-friendly meta analysis. Journal of Clinical Psychology, 65(5), 467-487. https://doi.org/10.1002/jclp.20593 
Sobel, M. E. (1982). Asymptotic confidence intervals for indirect effects in structural equation models. In S. Leinhardt (Ed.), Sociology Methodology 1982 (pp. 290-312). Washington DC: American Sociological Association. https://doi.org/10.2307/270723

Spielberger, C. D., Gorsuch, R. L., Lushene, R., Vagg, P. R., \& Jacobs, G. A. (1983). Manual for the State-Trait Anxiety Inventory. Palo Alto, CA: Consulting Psychologists Press.

Stevenson-Hinde, J. (2007). Attachment theory and John Bowlby: Some reflections. Attachment \& Human Development, 9(4), 337-342. https://doi.org/10.1080/14616730701711540

Tabachnik, B., \& Fidell, L. (2001). Using multivariate statistics. Boston: Pearson/Allyn \& Bacon.

Takebayashi, Y., Tanaka, K., Sugiura, Y., \& Sugiura, T. (2017). Well-Being and Generalized Anxiety in Japanese Undergraduates: A Prospective Cohort Study. Journal of Happiness Studies. https://doi.org/10.1007/s10902-017-9852-3

Weinstein, N., Brown, K., \& Ryan, R. (2009). A multi-method examination of the effects of mindfulness on stress attribution, coping, and emotional well-being. Journal Of Research In Personality, 43(3), 374-385. https://doi.org/10.1016/j.jrp.2008.12.008

Zaider, T., Heimberg, R., \& Iida, M. (2010). Anxiety disorders and intimate relationships: A study of daily processes in couples. Journal of Abnormal Psychology, 119(1), 163-173. https://doi.org/10.1037/a0018473

Zhang, X., Chen, X., Ran, G., \& Ma, Y. (2016). Adult children's support and self-esteem as mediators in the relationship between attachment and subjective well-being in older adults. Personality and Individual Differences, 97, 229-233. https://doi.org/10.1016/j.paid.2016.03.062

\section{Copyrights}

Copyright for this article is retained by the author(s), with first publication rights granted to the journal.

This is an open-access article distributed under the terms and conditions of the Creative Commons Attribution license (http://creativecommons.org/licenses/by/4.0/). 